\title{
La souffrance n'est pas une affaire médicale?
}

\author{
Hans Stalder \\ Prof. Dr méd., membre de la rédaction
}

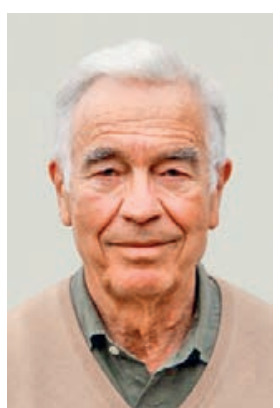

hans.stalder[at]saez.ch
The relief of suffering, it would appear, is considered one of the primary ends of medicine by patients and lay persons, but not by the medical profession [1]

La chambre médicale de la FMH a donc refusé de reprendre dans le code de déontologie la directive de l'Académie Suisse des Sciences Médicales "Attitude face à la fin de vie et à la mort». Ce texte propose le terme de souffrance intolérable comme critère d'entrée en discussion pour une assistance au suicide, remplaçant ainsi l'ancien, qui exige que «la maladie dont souffre le patient permet de considérer que la fin de la vie est proche». Est-ce que nous médecins n'avons plus l'habitude de considérer la souffrance quand nous sommes face à nos malades et préférons-nous des termes chiffrables? Nous mesurons effectivement les douleurs sur des échelles allant de 1 à 10 et traitons l'hypertension, le diabète et l'hyperlipémie comme des valeurs chiffrables en $\mathrm{mm} \mathrm{Hg}$ ou en $\mathrm{mmol} / \mathrm{l}$, selon des algorithmes souvent bien compliqués et parlons ensuite de non-compliance si ces valeurs ne sont pas atteintes.

Les patients qui nous consultent ne viennent pourtant pas pour quelques mmol ou mm Hg trop élevés, mais parce qu'ils souffrent. Et soyons conscients que beaucoup de nos interventions ne se basent pas sur des critères chiffrables mais sur la souffrance: nous remplaçons une tête de fémur parce que le patient trouve ses douleurs insupportables, traitons l'hypertrophie de la prostate parce que le patient ne supporte plus ses ennuis urinaires, dilatons une artère fémorale parce que le patient souffre de ne pas pouvoir marcher sans s'arrêter, et prescrivons des médicaments pour des hallucinations au moment où le patient ne les supporte plus.

Même si la souffrance est donc omniprésente c'est comme si ce terme nous faisait peur, et ce d'autant plus quand elle devient insupportable. Pas étonnant donc qu'à l'exception d'un excellent texte d'Eric J. Cassel apparu déjà en 1982 la littérature médicale ne déborde pas d'articles sur la souffrance. C'est seulement depuis 2002, quand la souffrance insupportable fut introduite explicitement dans les lois sur l'euthanasie en Hollande et en Belgique comme critère d'entrée en matière que le corps médical a recommencé à y réfléchir ([2,3], voir aussi [4]); c'est comme si la vox populi représentée par les parlementaires de ces pays avait forcé les médecins de s'en occuper à nouveau.

Comment alors définir la souffrance? Selon Cassel, "suffering is experienced by persons, not merely by bodies, and has its source in challenges that threaten the intactness of the person as a complex social and psychological entity" [1]. Souvent ce n'est même pas un problème de douleurs. Dans une étude qualitative chez des personnes traitées à domicile pour cancer demandant une euthanasie, faiblesse, fatigue, mal-être, dépendance et perte de contrôle sont plus fréquemment cités comme cause d'une souffrance intolérable que des problèmes médicaux [2]. Elle dépend en plus du passé de la personne, de la façon dont elle aborde les problèmes liés à la dégradation de son corps, de son expérience, de sa spiritualité et de sa culture ainsi que bien sûr de son environnement.

J'avoue que je suis un peu attristé que la chambre médicale a préféré un chiffre, par ailleurs pas très précis, à savoir le temps de vie encore présent, comme critère d'entrée en matière pour un suicide assisté plutôt que la souffrance, entité certes non mesurable, mais qui peut être transmise par le patient et ressentie par nous lors des entretiens approfondis et répétés pour autant que nous sachions l'entendre. Les Hollandais, Belges, Luxembourgeois et les citoyens de plusieurs Etats américains l'ont compris. Leurs médecins l'ont adopté, se sont investis, et dans l'ensemble ont fait de bonnes expériences. Pourquoi pas nous en Suisse? Faut-il aussi que le Parlement décide pour nous? Dans ces temps de digitalisation où l'on veut tout mesurer, ne devonsnous pas plutôt réapprendre à écouter le non-mesurable?

\section{Références}

1 Cassel EJ. The nature of suffering and the goals of medicine. N England J Med. 1982;306:639-45.

2 Dees MK, Vernooij-Dassen MJ, Dekkers WJ, Vissers KC, van Weel C. 'Unbearable suffering': a qualitative study on the perspectives of patients who request assistance in dying. Med Ethics. 2011;37:727-34.

3 Ruijs CDM, Kerkhof AJFM, van der Wal G, Onwuteaka-Philipsen BD. The broad spectrum of unbearable suffering in end-oflife cancer patients. A cross-sectional primary care study in the Netherlands. BMC Palliative Care. 2012;11:12.

4 Le numéro 1 du volume 11 (2018) de la revue de la Société Suisse d'Ethique Biomédicale Bioethica Forum est consacré à ce thème. 